\title{
Is Extra Mixing Really Needed in Asymptotic Giant Branch Stars?
}

\author{
Amanda I. Karakas \\ Research School of Astronomy \& Astrophysics, Mount Stromlo Observatory, Weston Creek ACT \\ 2611, Australia \\ akarakas@mso.anu .edu.au \\ Simon W. Campbell ${ }^{1,2}$ \\ Departament de Física i Enginyeria Nuclear, EUETIB, Universitat Politécnica de Catalunya, \\ C./Comte d'Urgell 187, E-08036 Barcelona, Spain \\ simon.w.campbell@upc.edu \\ and \\ Richard J. Stancliffe \\ Centre for Stellar $\&$ Planetary Astrophysics, Monash University, Clayton VIC 3800, Australia \\ Richard.Stancliffe@sci.monash.edu.au
}

\begin{abstract}
We demonstrate that the amount of extra mixing required to fit the observed low $\mathrm{C} / \mathrm{N}$ and ${ }^{12} \mathrm{C} /{ }^{13} \mathrm{C}$ ratios in first giant branch (FGB) stars is also sufficient to explain the carbon and nitrogen abundances of Galactic asymptotic/second giant branch (AGB) stars. We simulate the effect of extra mixing on the FGB by setting the composition of the envelope to that observed in low-mass $\left(M \leq 2 M_{\odot}\right)$ FGB stars, and then evolve the models to the tip of the AGB. The inclusion of FGB extra mixing compositional changes has a strong effect on the $\mathrm{C}$ and $\mathrm{N}$ abundance in our AGB models, leading to compositions consistent with those measured in Galactic carbon-rich stars. The composition of the models is also consistent with $\mathrm{C}$ and $\mathrm{N}$ abundances measured in mainstream silicon carbide $(\mathrm{SiC})$ grains. While our models cover the range of $\mathrm{C}$ abundances measured in carbon stars in the LMC cluster 1846, we cannot simultaneously match the composition of the $\mathrm{O}$ and $\mathrm{C}$-rich stars at the same time. A second important result is that our models only match the oxygen isotopic composition of $\mathrm{K}$ and some M, MS giants, and
\end{abstract}

\footnotetext{
${ }^{1}$ Institut de Ciéncies de l'Espai (CSIC), Campus UAB, Facultat de Ciéncies, Torre C5-parell, 2 planta, E-08193 Bellaterra (Barcelona), Spain

${ }^{2}$ Centre for Stellar \& Planetary Astrophysics, Monash University, Clayton VIC 3800, Australia
} 
are not able to match the oxygen composition of carbon-rich AGB stars. By increasing the abundance of ${ }^{16} \mathrm{O}$ in the intershell (based on observational evidence) it is possible to reproduce the observed trend of increasing ${ }^{16} \mathrm{O} /{ }^{18} \mathrm{O}$ and ${ }^{16} \mathrm{O} /{ }^{17} \mathrm{O}$ ratios with evolutionary phase. We also find that some Li production takes place during the AGB and that Li-rich carbon stars $(\log \epsilon(\mathrm{Li}) \gtrsim 1)$ can be produced. These models show a correlation between increasing $\mathrm{Li}$ abundances and $\mathrm{C}$. The models cannot explain the composition of the most Li-enriched carbon stars, nor can we produce a Li-rich carbon star if we assume extra mixing occurs during the FGB owing to ${ }^{3} \mathrm{He}$ destruction. We tentatively conclude that 1) if extra mixing occurs during the AGB it likely only occurs efficiently in low metallicity objects, or when the stars are heavily obscured making spectroscopic observations difficult, and 2) that the intershell compositions of AGB stars needs further investigation.

Subject headings: Galaxy: abundances - nuclear reactions, nucleosynthesis, abundances - stars: Abundances, stars: AGB and Post-AGB

\section{Introduction}

Standard stellar model 1 predict that there is only one mixing event that changes the surface composition between the main sequence and the tip of the first giant branch (FGB) - the first dredge-up (FDU). The models indicate that the FDU mixes the products of partial hydrogen burning into the envelope, with reductions in the ${ }^{12} \mathrm{C} /{ }^{13} \mathrm{C}$ ratio to $\sim 20$, and increases in ${ }^{14} \mathrm{~N}$ such that ${ }^{12} \mathrm{C} /{ }^{14} \mathrm{~N} \sim 1.5$. The ${ }^{16} \mathrm{O} /{ }^{17} \mathrm{O}$ ratio is reduced, depending on the mass of the star, whereas ${ }^{16} \mathrm{O} /{ }^{18} \mathrm{O}$ marginally increases (e.g., Boothrovd \& Sackmann 1999). Lithium, if not destroyed during the pre-main sequence, should be greatly reduced by the FDU. In contrast the envelope abundance of ${ }^{3} \mathrm{He}$, produced by the pp-chains during the main sequence, is significantly increased by the FDU. Observations, however, do not agree with these predictions. Low-mass giant stars have been found with ${ }^{12} \mathrm{C} /{ }^{13} \mathrm{C}$ ratios of $\sim 10$ and $\mathrm{C} / \mathrm{N} \sim 1.0$ (Gilrov 1989; Charbonnel 1994; Charbonnel et al. 1998). In globular clusters, the anticorrelation of $\mathrm{C}$ abundance with luminosity is also indicative of deep mixing occurring on the FGB (e.g., in M3; Smith 2002). Furthermore, chemical evolution models using standard ${ }^{3} \mathrm{He}$ yields (Dearborn et al. 1996; Romano et al. 2003) predict present-day abundances that are orders of magnitude greater than the values measured in meteorites and the local interstellar medium. These observations are used as evidence that some form of chemical transport (known variously as extra-mixing, deep mixing, or cool bottom processing) is acting in low-mass red giant envelopes.

\footnotetext{
${ }^{1}$ Standard in this context refers to models without non-convective extra mixing such as thermohaline mixing or rotation. This is distinguished from convective overshoot, which is also non-standard in stellar codes but is considered a natural extension of convective borders.
} 
In recent years there has been much effort devoted to finding the physical mechanism behind the extra mixing. Rotational mixing (Charbonnel et al. 1998), gravity waves (Denissenkov \& Tout 2000), thermohaline mixing (Eggleton et al. 2006, 2008; Charbonnel \& Zahn 2007; Stancliffe et al. 2009; Stancliffe 2010), and magnetic fields (Nordhaus et al. 2008; Busso et al. 2007; Palmerini et al. 2009) have been proposed as potential candidates. Parametric studies of extra mixing have also shown great promise in explaining the $\mathrm{C}, \mathrm{N}$, and $\mathrm{O}$ composition of first and asymptotic giant branch stars (Smith \& Tout 1992; Boothroyd \& Sackmann 1999; Nollett et al. 2003), although there are no compelling physical reasons for the choice of parameters that govern the mixing and burning.

The evidence that some form of non-convective mixing mechanism occurs during the AGB phase is more circumstantial. The argument put forward is similar to that for FGB giant stars. That is, the $\mathrm{C}$ and $\mathrm{N}$ abundances predicted by models do not match those observed in AGB stars (e.g., Lambert et al. 1986; Abia \& Isern 1997; Milam et al. 2009). In particular the ${ }^{12} \mathrm{C} /{ }^{13} \mathrm{C}$ ratios are lower than predicted by standard AGB models (e.g., Forestini \& Charbonnel 1997; Karakas 2010). Also, the $\mathrm{C}$ and $\mathrm{N}$ composition of pre-solar mainstream silicon carbide ( $\mathrm{SiC}$ ) grains, which are assumed to form in the extended envelopes of carbon-rich $(\mathrm{C} / \mathrm{O}>1)$ AGB stars, show a well defined distribution where $40 \lesssim{ }^{12} \mathrm{C} /{ }^{13} \mathrm{C} \lesssim 100$, and have nitrogen isotope ratios enhanced relative to the terrestrial value (e.g., Zinner 1998). In comparison, standard AGB models predict that by the time $\mathrm{C} / \mathrm{O} \geq 1$ the ${ }^{12} \mathrm{C} /{ }^{13} \mathrm{C} \geq 80$, which is already close to the upper limit observed in AGB stars or measured in $\mathrm{SiC}$ grains. A few studies have found that the only way to match observational data of carbon-rich AGB stars is to artificially lower the ${ }^{12} \mathrm{C} /{ }^{13} \mathrm{C}$ at the tip of the $\mathrm{FGB}$ to values observed in FGB stars (Kahane et al. 2000; Lebzelter et al. 2008). For example, by doing this Kahane et al. (2000) found the abundance ratios in their $2 M_{\odot}$ model of CW Leo were consistent with the observations. However, the authors did not go on to test this idea against data for a larger sample of Galactic carbon stars. Lithium is also enhanced in some carbon-rich AGB stars. The statistics indicate that super Li-rich stars with $\mathrm{Li}$ abundances as high as $\log \epsilon(\mathrm{Li}) \sim 52$ are rare, accounting for only a few percent of all carbon stars (Abia et al. 1993). However a larger fraction $(\sim 10 \%)$ are considered Li-rich, with $\mathrm{Li}$ abundances in the range 1-2 (although some J-stars are included in this fraction, Abia et al. 1993). Standard low-mass AGB models do not predict this variation in $\mathrm{Li}$, thus extra mixing may be needed to explain the high Li abundances in some AGB stars (Abia \& Isern 1997; Uttenthaler et al. 2008; Uttenthaler \& Lebzelter 2010). Intermediate-mass AGB stars can produce substantial quantities of $\mathrm{Li}$ via hot bottom burning (HBB; e.g., Boothroyd et al. 1993). While most C-rich stars will have a mass below the HBB limit $\left(M \lesssim 4 M_{\odot}\right)$, a few super-Li rich stars may have evolved from high mass AGB stars. These points are discussed further in $\oint 3$.

If we assume that thermohaline mixing (or some other mechanism) operates to change the envelope composition during the ascent of the FGB, then a natural questions arises: Do we need

\footnotetext{
${ }^{2}$ We use the standard notation $\log \epsilon(\mathrm{X})=\log _{10}\left(\mathrm{~N}_{\mathrm{X}} / \mathrm{N}_{\mathrm{H}}\right)+12.0$, where $N_{\mathrm{X}}$ is the number abundance of species $\mathrm{X}$ and $N_{\mathrm{H}}$ is the number abundance of hydrogen.
} 
further extra mixing on the AGB to be able to match the observed data? It is this question that we seek to address here. This question is particularly timely, given the recent efforts to find the physical mechanism driving the extra mixing on the FGB (e.g., Eggleton et al. 2008). In this paper we argue that there is no strong need for extra mixing on the AGB, at least for Galactic carbon stars of near solar metallicity. For the moderately metal-poor AGB stars in the LMC and SMC the situation is less clear (see also the discussion in Lederer et al. 2009).

In the current study we evolve a series of low-mass AGB models, some of which we assume have experienced extra mixing on the FGB. We then compare our models with observations of $\mathrm{C} / \mathrm{O}$ ratios and carbon isotopic data in a sample of Galactic carbon stars and mainstream $\mathrm{SiC}$ grains, and the compositions of stars in the Magellanic Clusters NGC 1846 and NGC 1978. We include a discussion of lithium and the oxygen isotope ratios in AGB stars, as these species may elucidate the occurrence and nature of possible extra mixing in AGB stars.

\section{Stellar Models}

We evolve $1.25 M_{\odot}$ and $1.8 M_{\odot}, Z=0.01$ stellar models from the zero age main sequence (ZAMS) to the tip of the AGB. These masses were chosen because the $1.25 M_{\odot}$ is likely to be close to the lower mass limit for carbon star formation, whereas the $1.8 M_{\odot}$ is a typical carbon star mass in

both the Galaxy (Wallerstein \& Knapp 1998; Abia et al. 2001) and in the LMC (Lebzelter \& Wood 2007). In regard to the metallicities of Galactic carbon stars, Lambert et al. (1986) noted that $-0.3<[\mathrm{M} / \mathrm{H}]<0.0$, where $\mathrm{M}$ is the total metal abundance. These results were confirmed by Abia et al. (2002) who confirm that the metallicities of N-type carbon stars are of nearly solar metallicity. These results suggest our choice of $Z=0.01$ (or $[\mathrm{Fe} / \mathrm{H}]=-0.15)$ is consistent with the metallicities of Galactic carbon stars. We calculate the structure first and perform detailed nucleosynthesis calculations afterward, using a post-processing algorithm. The details of this procedure and the codes used to compute the models have been previously described in detail (e.g., Karakas et al. 2009). In the nucleosynthesis step we use 77 species, and a scaled-solar initial composition where we take the solar abundances from Asplund et al. (2005) and assume $Z_{\text {solar }}=0.015$. Mass loss on the FGB is included using the Reimer's formula with the parameter $\eta=0.4$ and on the AGB we use the Vassiliadis \& Wood (1993, hereafter VW93) mass-loss prescription. The VW93 mass-loss prescription keeps the mass-loss rate relatively low until the beginning of the superwind phase, at which point the mass-loss rate dramatically increases to a few $\times 10^{-5} M_{\odot}$ year ${ }^{-1}$. This has the effect of keeping the envelope mass relatively high, compared to using the Reimer's massloss rate on the AGB, and may affect the depth of third dredge-up (TDU). Stancliffe \& Jeffery (2007) discuss the effect of varying the mass loss on the evolution and nucleosynthesis of a $1.5 M_{\odot}$, $Z=0.008$ model. They find that the total amount of material that gets dredged-up to be similar for all three mass-loss prescriptions (VW93, Reimer's with $\eta=1$, and Blöcker 1995, with $\eta=0.02$ ). The surface $\mathrm{C} / \mathrm{O}$ ratio, however, tended to be higher at a given time in the Reimer's model than in the other two cases. The reason given for this is because models using Reimer's law typically had 
a small envelope mass at a given thermal pulse number, It should be noted that both Reimer's and the Blöcker mass loss critically depend on the choice of the free parameter $\eta$, the values of which are uncertain.

The main changes to the stellar structure code that have been implemented for this study are the inclusion of the C and N-rich low temperature opacity tables from Lederer \& Aringer (2009), and the inclusion of convective overshoot to induce the TDU in low-mass models. The TDU is the inward movement of the convective envelope into regions processed by partial He-burning during a thermal instability (see Herwig 2005, for a recent review). Some form of overshoot is required because low-mass models computed previously with the same codes show little or no TDU for masses $\lesssim 2 M_{\odot}$ at near solar metallicities (Karakas et al. 2002). Other stellar evolution codes also show no TDU at these masses without some form of convective overshoot (Mowlavi 1999; Herwig] 2000). We include overshoot following a thermal pulse by extending the position of the base of the convective envelope by $N_{\text {ov }}$ pressure-scale heights. To obtain a carbon-rich envelope we require $0.5 \lesssim N_{\text {ov }} \lesssim 5$, depending on the mass of the model. In the $1.25 M_{\odot}$ model we set $N_{\text {ov }}=3,4$, and in the $1.8 M_{\odot}$ model we set $N_{\mathrm{ov}}=1,2,3$. Experimenting with the efficiency of overshoot in AGB models is reasonable, given our lack of understanding of the physics of convection in stars. The location of the inner edge of the convective envelope has been shown to depend critically on the numerical details of a particular stellar evolution code (see Fig. 1 in Frost \& Lattanzio 1996), and different stellar evolution codes predict dissimilar results for the same masses and metallicities (e.g., Straniero et al. 1997; Mowlavi 1999; Karakas et al. 2002; Stancliffe et al. 2004). Efforts to match the carbon-star luminosity function in the Magellanic Clouds typically assume more efficient TDU than found in low-mass stellar models (e.g., Marigo et al. 1999, but see also Stancliffe et al. 2005.) Furthermore, it is still unknown if the efficiency of TDU is stochastic in nature, or there is one depth, for a given total stellar mass, H-exhausted core mass, and composition.

In Table 1 we show the initial, post-FDU, tip of the FGB for the extra-mixing case ${ }^{3}$, and tip of the AGB ratios for $\mathrm{C} / \mathrm{O},{ }^{12} \mathrm{C} /{ }^{13} \mathrm{C},{ }^{12} \mathrm{C} /{ }^{14} \mathrm{~N},{ }^{14} \mathrm{~N} /{ }^{15} \mathrm{~N},{ }^{16} \mathrm{O} /{ }^{17} \mathrm{O}$, and ${ }^{16} \mathrm{O} /{ }^{18} \mathrm{O}$ from the stellar models. The post-FDU values of ${ }^{12} \mathrm{C} /{ }^{13} \mathrm{C}$ and $\mathrm{C} / \mathrm{N}$ for the $1.25 M_{\odot}$ model are 27 and 1.48 , respectively. The post-FDU values for the $1.8 M_{\odot}$ model are 22 and 1.0. These numbers are consistent with other standard model predictions (El Eid 1994; Charbonnel 1994; Boothroyd \& Sackmann 1999).

To simulate the effect of extra mixing on the FGB, we take the envelope composition at the tip of the giant branch and alter it such that the ${ }^{12} \mathrm{C} /{ }^{13} \mathrm{C}$ ratio equals 10 (from Fig. 6 of Charbonnel 1994), and the $\mathrm{C} / \mathrm{N}$ ratio $\approx 1$ (Table 1 in Gilroy \& Brown 1991). This is done by decreasing the ${ }^{12} \mathrm{C}$ abundance and increasing the abundances of ${ }^{13} \mathrm{C}$ and ${ }^{14} \mathrm{~N}$, as expected from $\mathrm{CN}$ cycling. The composition of the oxygen isotopes are not altered because the observed data for FGB stars are consistent with model predictions (Dearborn 1992; Boothroyd \& Sackmann 1999). By using the lower limit for ${ }^{12} \mathrm{C} /{ }^{13} \mathrm{C}$ from the observations (which show values between 10 and 18 ) we expect to maximize the effect on the final AGB composition. We would expect intermediate values to give

\footnotetext{
${ }^{3}$ for the models without extra mixing, the abundances at the tip of the FGB are the same as after the FDU.
} 
results that fall between our extreme case and the no mixing model. For the $1.8 M_{\odot}$ case the $\mathrm{C} / \mathrm{N}$ ratio is already 1.0 after FDU. After "extra-mixing", a reduction in ${ }^{12} \mathrm{C}$ and increases in ${ }^{13} \mathrm{C}$ and ${ }^{14} \mathrm{~N}$ result in $\mathrm{C} / \mathrm{N} \approx 0.9$ (see Table 1). This value is still within the range of observed $\mathrm{C} / \mathrm{N}$ ratios observed in FGB stars, that show $\mathrm{C} / \mathrm{N}$ ratios between $\sim 0.8$ to 1.2 (Gilroy \& Brown 1991).

Along with simulating the effect of extra mixing on the composition of the envelope, we perform two further tests. The first is to increase the ${ }^{16} \mathrm{O}$ abundance at the ZAMS so that the initial $\mathrm{C} / \mathrm{O}$ ratio $=0.35$ (in comparison to other models which have a solar $\mathrm{C} / \mathrm{O}=0.5$ ). This is equivalent to an $\alpha$-enhanced composition, where $[\mathrm{O} / \mathrm{Fe}] \approx+0.20$. We do this because $\mathrm{F}$ and $\mathrm{G}$ stars in our Galaxy show moderate enhancements of $\alpha$ elements (e.g., Reddy et al. 2003), and perhaps some of the Galactic AGB stars have similar moderate enhancements.

The second test is to increase the intershell abundance of ${ }^{12} \mathrm{C}$ and ${ }^{16} \mathrm{O}$ to values observed in the central stars of planetary nebulae. Standard model predictions provide values of about $25 \%$ (by mass) of ${ }^{12} \mathrm{C}$ in the intershell, and only $\lesssim 2 \%$ of ${ }^{16} \mathrm{O}$. Observations of post-AGB central stars suggest the intershell abundances are between $2-20 \%$ for ${ }^{16} \mathrm{O}$ and $15-60 \%$ for ${ }^{12} \mathrm{C}$ (Werner et al. 2009).

\section{Model predictions versus real stars}

In Fig. 11 we show the distribution of ${ }^{12} \mathrm{C} /{ }^{13} \mathrm{C}$ ratios from Lambert et al. (1986). The sample average ${ }^{12} \mathrm{C} /{ }^{13} \mathrm{C}$ ratio for the C-rich stars is 58, excluding the J-stars, or 50 with the J-stars included. The standard deviation on these numbers are 25 and 17, with and without the J-stars, respectively. We choose to use this data-set for comparison because these authors use infra-red observations to derive the ${ }^{12} \mathrm{C} /{ }^{13} \mathrm{C}$ ratios and are likely more reliable for the very red, cool carbonrich $\mathrm{AGB}$ stars under consideration here. We exclude the ${ }^{12} \mathrm{C} /{ }^{13} \mathrm{C}$ ratios for the J-type carbon stars, which have carbon isotope ratios close to the equilibrium value of $\approx 4$. The evolutionary origin of these ${ }^{13} \mathrm{C}$-rich stars has not been determined. Many J-stars do not show enrichments in $s$-process elements, in contrast to $\mathrm{N}$-type stars and it is questionable if they are really TP-AGB stars (Wallerstein \& Knapp 1998). The average value of the ${ }^{12} \mathrm{C} /{ }^{13} \mathrm{C}$ ratio for mainstream $\mathrm{SiC}$ grains is 57 with a standard deviation $\sigma=16$ (using data from Gyngard et al. 2006), consistent with the average value derived for the C-rich stars in Fig. 1.

Included in Fig. 1 are model predictions from the $1.25 M_{\odot}$ (top panel), and the $1.8 M_{\odot}$ (lower panel) AGB models. In the top panel, the dashed vertical line shows the ${ }^{12} \mathrm{C} /{ }^{13} \mathrm{C}$ ratio at the tip of the AGB for the $N_{\mathrm{ov}}=3$ model with no extra mixing on the FGB, whereas the dot-dashed vertical line shows the result when the ${ }^{12} \mathrm{C} /{ }^{13} \mathrm{C}$ ratio is reduced at the tip of the FGB in line with observations. This clearly shows the strong effect on the carbon isotope ratio in AGB stars when taking FGB mixing into account. In this example the ${ }^{12} \mathrm{C} /{ }^{13} \mathrm{C}$ ratio is reduced from $\sim 76$ to 33 . The dotted vertical line shows the ${ }^{12} \mathrm{C} /{ }^{13} \mathrm{C}$ ratio for the $N_{\text {ov }}=4$ case with extra mixing on the FGB. This shows the effect of deeper TDU increasing the isotopic ratio due to the extra ${ }^{12} \mathrm{C}$ that 
is dredged up. Likewise for the bottom panel the dashed vertical line shows the ${ }^{12} \mathrm{C} /{ }^{13} \mathrm{C}$ ratio at the tip of the AGB for the $N_{\text {ov }}=1$ model with no extra mixing, whereas the results including extra mixing are shown by the vertical dot-dashed line. Again, we can see the significant effect of including the FGB abundance ratios. The dotted vertical line shows the ${ }^{12} \mathrm{C} /{ }^{13} \mathrm{C}$ ratio for the $N_{\text {ov }}=2$ case with extra mixing (without FGB extra mixing this model has a ${ }^{12} \mathrm{C} /{ }^{13} \mathrm{C}$ ratio $>100$, off the graph). In summary, from Fig. 1 we see that the models considered here span the observed range of ${ }^{12} \mathrm{C} /{ }^{13} \mathrm{C}$ ratios, with the exception of one object which has a ratio of $19 \pm 6$. Two objects have ${ }^{12} \mathrm{C} /{ }^{13} \mathrm{C}$ ratios $\approx 30 \pm 5$ (Lambert et al. 1986) but the uncertainties $( \pm 5)$ allow these two stars to be spanned by the model predictions. Furthermore, it is possible that not all C-stars will be at the tip of the AGB. Some of the carbon stars may have a value between the FGB tip (after modification by extra mixing) and the AGB tip value.

In Fig. 2 we illustrate the temporal evolution of the $\mathrm{C} / \mathrm{O}$ ratio versus the ${ }^{12} \mathrm{C} /{ }^{13} \mathrm{C}$ ratio for the $1.8 M_{\odot}$ model with $N_{\text {ov }}=3$ (open symbols and lines, refer to the caption for details). Note that each symbol represents the model abundances after a TDU episode. This model was chosen because it reaches a high final $\mathrm{C} / \mathrm{O}$ ratio of 3.3 (models with different values of $N_{\text {ov }}$ follow similar tracks, but finish at lower $\mathrm{C} / \mathrm{O}$ ratios). It will become apparent that once we start enhancing the $\mathrm{O}$ abundance (either at the beginning of the evolution, or in the intershell) the final $\mathrm{C} / \mathrm{O}$ ratios will be reduced and for this comparison we require the final $\mathrm{C} / \mathrm{O}$ ratio $>1$. In Fig. 2 we show results from models with scaled-solar and $\alpha$-enhanced compositions, with and without extra mixing on the FGB. The labels on each line show the $\mathrm{C} / \mathrm{O}$ and ${ }^{12} \mathrm{C} /{ }^{13} \mathrm{C}$ ratios at the tip of the FGB.

Note that the models with an $\alpha$-enhanced composition (i.e., lower initial $\mathrm{C} / \mathrm{O}$ ratio) shift the predictions to the left, and result in a steeper slope and lower final $\mathrm{C} / \mathrm{O}$ ratio. Also included in Fig. 2 are observational data for Galactic carbon stars from Lambert et al. (1986), and stars in the Magellanic Cloud clusters NGC 1846 and NGC 1978 using data from Lebzelter et al. (2008) and Lederer et al. (2009), respectively. These clusters have a lower metallicity $([\mathrm{Fe} / \mathrm{H}] \sim-0.5)$ than considered here $([\mathrm{Fe} / \mathrm{H}] \sim-0.15)$, and the AGB mass of NGC 1978 is smaller, at $1.5 M_{\odot}$ (Lederer et al. 2009). It is worth noting that the abundance tracks of the $1.25 M_{\odot}$ model (not shown) follow almost the same path as the $1.8 M_{\odot}$ models, the only noticeable difference being a final lower $\mathrm{C} / \mathrm{O}$ ratio as a consequence of fewer thermal pulses and TDU episodes. Even so, it is clear from Fig. 2 that the various stellar models cover most of the range of observational data points. This agreement is especially encouraging considering that we are using only one mass and one metallicity to cover the range of observed carbon-rich AGB stars, where there is likely a spread in initial mass, composition, and evolutionary state.

The hypothesis put forward by Lebzelter et al. (2008) to explain the composition of the NGC 1846 stars is as follows. The O-rich stars were matched by assuming extra mixing on the FGB plus an $\alpha$-enhanced composition $([\mathrm{O} / \mathrm{Fe}]=+0.2)$, such that the $\mathrm{C} / \mathrm{O}$ ratio $=0.2$ at the tip of the $\mathrm{FGB}$, consistent with the observations which yield $\mathrm{C} / \mathrm{O}=0.2 \pm 0.05$. To explain the composition of the two carbon-rich NGC 1846 stars near the bottom right of Fig. 2 required extra mixing on the FGB and AGB, but mixing only occurs on the AGB after the stars had become carbon rich. 
It is not easy to understand why mixing would only start once $\mathrm{C} / \mathrm{O}>1$ in the envelope, given that there are several episodes of TDU before this. While the outer envelope (e.g., the opacities) strongly depend on the composition, the structure of the star near the inner region of the envelope and the H-shell do not. There are no noticeable changes to the structure of the burning shells, nor in the time between pulses as the star evolves from $\mathrm{C} / \mathrm{O}<1$ to carbon rich. Fig. 2 shows that our scaled-solar models with FGB extra mixing alone can explain the composition of these two C-rich stars. However, the models that fit the two stars have a higher (0.32) C/O ratio at the tip of the FGB than observed in the O-rich stars by Lebzelter et al. (2008). Hence our models cannot simultaneously fit the composition of the $\mathrm{O}$ and $\mathrm{C}$-rich stars at the same time. One test of the AGB extra mixing scenario would be the determination of the Li abundances in the two stars. A high Li abundance may imply that these stars have experienced extra mixing on the AGB whereas a low Li abundance may rule out the need for extra mixing.

Lastly in this section we discuss the results for lithium from our stellar models. We will use the $1.8 M_{\odot}$ model with $N_{\text {ov }}=3$ as an example. While some $\mathrm{C}$ and Li-rich stars with $\mathrm{C} / \mathrm{O}$ ratios $\approx 1$ may have evolved from high-mass AGB stars undergoing HBB, most C-rich objects evolved from lower mass stars. The first dredge-up reduces the ${ }^{7} \mathrm{Li}$ envelope abundance from $\log \epsilon(\mathrm{Li})$ from 3.14 at the ZAMS to 1.28. It is likely that our models do not suffer enough Li depletion from the first dredge-up, where observations of $\mathrm{G}-\mathrm{K}$ giants suggest most stars have $\log \epsilon(\mathrm{Li})<1.0$ (Brown et al. 1989). During the TP-AGB the ${ }^{7} \mathrm{Li}$ abundance in our models increases as a consequence of the $\mathrm{TDU}$, and reaches a maximum of $\log \epsilon(\mathrm{Li})=1.80$. The increase of $\mathrm{Li}$ mostly takes place before efficient TDU begins to penetrate into the He-intershell (i.e., during the first few TPs which are weak), but after this we find a correlation between the Li abundance and carbon (and presumably, $s$-process elements). This could explain the correlation found between Li and Tc abundances by Vanture et al. (2007) and Uttenthaler \& Lebzelter (2010). We note that the final Li abundance of this model would be considered Li-rich according to the definition of Abia et al. (1993) i.e., $\log \epsilon(\mathrm{Li}) \geq 1$.0. If our model had suffered extra depletion on the FGB, then it is possible that the $\mathrm{Li}$ abundance would not have reached $\log \epsilon(\mathrm{Li}) \geq 1.0$ during the TP-AGB. We test this by reducing the envelope ${ }^{7} \mathrm{Li}$ abundance at the tip of the FGB to $\log \epsilon(\mathrm{Li})=0.0$ (close to the center of the $\mathrm{Li}$ abundance distribution for G-K giants, Fig. 3 from Abia et al. 1993). We also reduce the envelope ${ }^{3} \mathrm{He}$ abundance by a factor of 10 , because this isotope would also be destroyed by whatever process destroys the lithium. While we find a similar trend as before, with Li increasing prior to the start of deep TDU and then slowly thereafter, the final value is 0.76 , less than the minimum for Li-rich carbon stars.

\subsection{The oxygen isotope ratios}

In this section we compare our models to oxygen isotopic measurements of FGB and AGB stars. In particular, we are interested to see if standard AGB models can match the observed composition, and if not, do we need to resort to extra mixing on the AGB? We focus on oxygen 
isotopic data from the stellar spectra of evolved stars and do not compare to the oxygen composition of presolar oxide grains. We do this because the oxygen isotope data for stars spans a smaller range than measured in grains, with few stars found in the region occupied by Group 2 grains, and no stars found in the region occupied by Groups 3 and 4 (Fig. 9 from Nittler et al. 1997). If these grains formed in the outflows of AGB stars then there should be stellar counterparts. Or perhaps the grains formed in circumstellar envelopes around AGB stars much more evolved (and heavily obscured) than the MS, S and C-type giants observed by Harris and collaborators. If so, it is curious that the $\mathrm{C}$ isotope data measured in $\mathrm{SiC}$ grains span the same range as measured in $\mathrm{AGB}$ stars while the $\mathrm{O}$ isotopic data in oxide grains do not.

In Fig. 3 we show the ${ }^{16} \mathrm{O} /{ }^{18} \mathrm{O}$ ratio versus the ${ }^{16} \mathrm{O} /{ }^{17} \mathrm{O}$ ratio from a sample of $\mathrm{G}$ and $\mathrm{K}$ giants, barium stars, and carbon-rich AGB stars. References are provided in the figure caption. Error bars are not included but uncertainties are large; we refer to Harris et al. (1987) for a detailed discussion. Included in the figure are our predictions from the $1.25 M_{\odot}$ and $1.8 M_{\odot}$ scaled-solar models. We note that we are using models without extra mixing on the FGB, but the results would not change because we assume that the oxygen isotope ratios are not altered by this mixing. We also indicate in the figure the solar oxygen isotope ratios, which provide the starting position for our models (the dotted lines). In Table 1 and Fig. 3, it can be seen that the FDU reduces the ${ }^{16} \mathrm{O} /{ }^{17} \mathrm{O}$ ratio to $\sim 330$ in the $1.8 M_{\odot}$ model, and 1800 in the $1.25 M_{\odot}$ case, as a consequence of CNO cycling that synthesizes some ${ }^{17} \mathrm{O}$. In contrast, the ${ }^{16} \mathrm{O} /{ }^{18} \mathrm{O}$ ratio increases slightly as a consequence of ${ }^{18} \mathrm{O}$ destruction. These numbers are reasonably consistent with the $\mathrm{O}$ isotope ratios measured in FGB stars (Harris \& Lambert 1984).

It is also evident in Table 1 and Fig. 3 that the predicted oxygen isotope ratios at the tip of the AGB do not match the isotopes measured in C-rich AGB stars. In particular, the oxygen isotope ratios derived from observations of AGB stars are notably larger than the model predictions. Is this caused by AGB extra mixing? This is unlikely, as the changes caused by extra mixing would shift the ${ }^{16} \mathrm{O} /{ }^{17} \mathrm{O}$ and ${ }^{16} \mathrm{O} /{ }^{18} \mathrm{O}$ ratios in a similar direction as the FDU. That is, the abundance of ${ }^{17} \mathrm{O}$ would be enhanced, ${ }^{18} \mathrm{O}$ destroyed, and ${ }^{16} \mathrm{O}$ would remain largely unchanged. This would result in increases in ${ }^{16} \mathrm{O} /{ }^{18} \mathrm{O}$ and decreases in ${ }^{16} \mathrm{O} /{ }^{17} \mathrm{O}$. Instead, both ratios appear to increase in the stars with evolution. Herwig (2000) also noted that the oxygen isotope ratios measured in AGB stars are correlated with each other, and seem to be correlated with the observed carbon and $s$-process element abundances. Hence it is likely that the increase of the oxygen isotope ratios with evolutionary state can be attributed to the TDU.

We now explore another possibility to explain the discrepancy. The operation of the TDU mixes material from the He-burning shell, that has been exposed to partial He-burning, to the surface. The composition of the intershell is mostly ${ }^{4} \mathrm{He}$ and ${ }^{12} \mathrm{C}$ (with typical compositions $75 \%$ and $25 \%$, respectively). Partial He-burning also synthesizes small quantities of ${ }^{16} \mathrm{O}$ and ${ }^{22} \mathrm{Ne}$, at the level of a few percent each at most. Could it be that the amount of ${ }^{16} \mathrm{O}$ in the intershell is higher than predicted by standard models? 
Support for this idea comes from both observational and theoretical fronts. The composition of hot post-AGB stars show $\mathrm{C}$ abundances between 15-60\%, O abundances between 2-20\%, and Ne abundances of about 2\% (De Marco \& Barlow 2001; Wesson et al. 2008; Werner et al. 2009). The theoretical models of Herwig (2000) with exponentially decaying overshoot at all convective borders find $\mathrm{O}$ abundances in the intershell of $\sim 20 \%$. The overshoot penetrates into the top of the $\mathrm{C}-\mathrm{O}$ core, increasing the $\mathrm{C}$ and $\mathrm{O}$ content of the intershell during a convective thermal pulse. Furthermore, key He-burning reactions rates (e.g., ${ }^{12} \mathrm{C}(\alpha, \gamma){ }^{16} \mathrm{O}$ ) are uncertain and could allow for higher ${ }^{16} \mathrm{O}$ intershell abundances.

The next question is how do these intershell compositions effect the envelope composition? To test this idea, we synthetically compute the envelope abundances of the $1.25 M_{\odot}$ model with $N_{\mathrm{ov}}=4$, and $1.8 M_{\odot}$ model with $N_{\mathrm{ov}}=3$. All tests assume a scaled-solar initial composition and that extra mixing has taken place on the FGB. From the detailed stellar structure computations, we have the total mass, H-exhausted core mass, and mass dredged into the envelope as a function of thermal pulse number. Assuming a given set of intershell abundances we then calculate the envelope abundance as a function of pulse number. In Table 2 we show the ${ }^{12} \mathrm{C}$ and ${ }^{16} \mathrm{O}$ intershell abundances used (in mass fractions), and the resultant surface abundances at the tip of the AGB for the $\mathrm{C} / \mathrm{O},{ }^{12} \mathrm{C} /{ }^{13} \mathrm{C},{ }^{16} \mathrm{O} /{ }^{17} \mathrm{O}$, and ${ }^{16} \mathrm{O} /{ }^{18} \mathrm{O}$ ratios. The surface nitrogen isotope ratio is assumed not to change as a consequence of increased oxygen in the intershell. These results are illustrated in Fig. 3 for the oxygen isotope ratios, and in Fig. 4 for the $\mathrm{C} / \mathrm{O}$ and ${ }^{12} \mathrm{C} /{ }^{13} \mathrm{C}$ ratios. In Fig. 3 the open squares show the result of using an intershell composition of $20 \%$ for $\mathrm{O}$ in the $1.8 M_{\odot}$ model. Note that the oxygen isotope ratios do not significantly shift from the post-FDU values for the $1.8 M_{\odot}$ model assuming a standard intershell composition. The open circles show the evolution of the $\mathrm{O}$ isotope ratios in the $1.25 M_{\odot}$ case with a standard intershell composition $(26 \% \mathrm{C}$ and $0.08 \%$ O), whereas the open triangles show the result of using $20 \%$ O. The two models with O-rich intershells show a similar trend of increasing oxygen isotope ratios with evolution as seen in the observations.

The comparison is not perfect, however, but the tests performed here are simple and rely on many assumptions. First, we have assumed constant intershell abundances, whereas detailed models show that the He-intershell abundances of ${ }^{12} \mathrm{C}$ and ${ }^{16} \mathrm{O}$ depend on the total mass, core mass, initial composition, and time during the convective thermal pulse. Second, we have only enhanced the abundance of ${ }^{12} \mathrm{C}$ and ${ }^{16} \mathrm{O}$, and ignored changes to the minor ${ }^{17} \mathrm{O}$ and ${ }^{18} \mathrm{O}$ isotopes. This is a valid assumption given that the $\mathrm{C}-\mathrm{O}$ core below the He shell contains only trace quantities of these isotopes. Also, we have only considered models of two masses, whereas real carbon-rich AGB stars in the Galaxy likely evolve from initial masses between about $1.2 M_{\odot}$ to $\sim 3 M_{\odot}$. Certainly models of stars of mass between $1.25 M_{\odot}$ and $1.8 M_{\odot}$ may help fill in the gap between the model predictions in Fig. 3. We also do not vary the rates of the uncertain ${ }^{17} \mathrm{O}(p, \gamma){ }^{18} \mathrm{~F}$ and ${ }^{17} \mathrm{O}(p, \alpha){ }^{14} \mathrm{~N}$ reactions, which play an important role in setting the minimum ${ }^{16} \mathrm{O} /{ }^{17} \mathrm{O}$ value reached after the FDU. The new evaluations of the ${ }^{17} \mathrm{O}+p$ rates by Chafa et al. (2007) have reduced uncertainties compared to previous estimates, but these still range between $\sim 20 \%$ to a factor of two, depending 
on temperature.

In Fig. 4 we show the results of an increasing $\mathrm{C}$ and $\mathrm{O}$ intershell composition on the evolution of the $\mathrm{C} / \mathrm{O}$ and ${ }^{12} \mathrm{C} /{ }^{13} \mathrm{C}$ ratios from the $1.8 M_{\odot}$ model with $N_{\text {ov }}=3$. The solid line shows model predictions assuming a standard intershell composition $(24 \% \mathrm{C}$ and $0.6 \% \mathrm{O})$ and extra mixing on the FGB. The dashed and dot-dashed lines show the result of increasing the ${ }^{16} \mathrm{O}$ content of the intershell to $10 \%$ (dashed) and $15 \%$ (dot-dashed), which has the effect of shifting the final $\mathrm{C} / \mathrm{O}$ ratio to lower values. We note that the extra ${ }^{16} \mathrm{O}$ from the intershell helps to keep the final $\mathrm{C} / \mathrm{O}$ ratio within the observed range. The dotted line illustrates results that use the composition of PG 1159-035, which has 48\% C and 17\% O (Jahn et al. 2007; Wesson et al. 2008), in the intershell. That the model predictions in Fig. 4 fit the data for Galactic carbon stars remarkably well may indicate that current AGB models predict too little ${ }^{16} \mathrm{O}$ in the intershell. This uncertainty warrants further investigation.

\section{Discussion and concluding remarks}

We now return to the original question posed in the Introduction: Do we need extra mixing on the AGB? If we simply seek to explain the $\mathrm{C} / \mathrm{O}$ and ${ }^{12} \mathrm{C} /{ }^{13} \mathrm{C}$ ratios in Galactic AGB stars then we have found the answer to be no. The inclusion of extra mixing on the FGB followed by a standard thermally-pulsing AGB evolution can produce $\mathrm{C}$ and $\mathrm{N}$ compositions consistent with values observed in AGB stars and measured in pre-solar $\mathrm{SiC}$ grains.

But what about Li in carbon-rich Galactic AGB stars? The existence of stars that are both C- and Li-rich has led some authors to conclude that extra mixing occurs in some fraction of AGB stars (e.g., Abia \& Isern 1997). The frequency of carbon stars rich in Li is $\lesssim 10 \%$, implying that the majority of carbon stars are $\mathrm{Li}$ normal or $\mathrm{Li}$ poor $(\log \epsilon(\mathrm{Li})<1)$. Furthermore, there is evidence that the Li production is tied to thermal pulses (and possibly the TDU) in AGB stars, as indicated by the correlation between AGB stars rich in Li and enriched in the unstable $s$-process element Tc (Vanture et al. 2007; Uttenthaler \& Lebzelter 2010). We found that lithium is produced during the TP-AGB phase of the $1.8 M_{\odot}$ model with deep TDU, and we find a correlation between increasing $\mathrm{Li}$ and carbon in the envelope (and presumably also $s$-process elements). That our models can reproduce the correlation between He-shell products and Li found by Uttenthaler \& Lebzelter (2010) is compelling, but further study into Li nucleosynthesis is required. Our model can only become a Li-rich carbon star when we assume that no extra mixing has taken place during the FGB. This model then has a higher ${ }^{12} \mathrm{C} /{ }^{13} \mathrm{C}$ and $\mathrm{C} / \mathrm{O}$ ratio than observed. If we assume extra mixing has taken place on the FGB, the star still produces Li but is no longer considered "Li-rich", hence extra mixing would be required during the AGB to provide the Li. However we restate that only $\sim 10 \%$ of carbon stars are observed to be Li-rich, which indicates that efficient extra mixing during the AGB is not required in the majority of these objects. We note that this is consistent with our findings for $\mathrm{C}$ and $\mathrm{N}$. The spread in Li observed in carbon-rich AGB stars may be explained by the fact we have used one stellar mass and metallicity for our tests whereas Galactic carbon stars will have 
a range of stellar masses, some of which are predicted not to experience any extra mixing during the FGB (i.e., $M \geq 2 M_{\odot}$ Eggleton et al. 2008). Also, it is possible that stars experience some variation in the mixing efficiency, with the Li abundance able to increase or decrease depending on the details of the mixing and burning (Wasserburg et al. 1995).

The low $\mathrm{C} / \mathrm{O}$ ratios present in C-rich AGB stars as compared to theoretical models (see e.g., Fig. 2) have been previously explained by allowing for extra mixing on the AGB or by the use of Crich low-temperature opacities. Extra mixing would reduce the $\mathrm{C}$ abundance but keep the elemental O abundance approximately constant (e.g., Nollett et al. 2003). Marigo (2002) showed that the use of C-rich low-temperature opacities leads to an increase of the mass-loss rate and therefore limits the final $\mathrm{C} / \mathrm{O}$ of the model star. In our detailed models we use the latest low-temperature C-rich opacities from Lederer \& Aringer (2009) and we still find higher final C/O ratios than spanned by the observations, although these models depend on the assumed efficiency of convective overshoot. Even so, our models suggest that there are only two ways to reduce the $\mathrm{C} / \mathrm{O}$ ratio in AGB models: Variations in the intershell abundances or extra mixing on the AGB. In Fig. 4 we show that models with high $\mathrm{O}$ intershell abundances are able to produce $\mathrm{C} / \mathrm{O}$ ratios within the range measured in Galactic carbon stars. With a reduced TDU, these same models may also be able to account for AGB stars rich in $s$-process elements but with spectral types $\mathrm{M}$ and $\mathrm{MS}$, which have $\mathrm{C} / \mathrm{O}<1$. We have shown extra mixing need not be the only explanation for these observations. This was also discussed by Herwig (2000) although his models were more massive $\left(3,4 M_{\odot}\right)$ than considered here and likely more massive than most carbon stars.

The case for extra mixing on the AGB is much stronger at low metallicity. The LMC clusters NGC 1846 and NGC 1978 both have a metallicity lower than solar, although NGC 1846 is slightly more metal poor with $[\mathrm{Fe} / \mathrm{H}]=-0.49$ (Grocholski et al. 2006), whereas NGC 1978 has $[\mathrm{Fe} / \mathrm{H}]$ $=-0.37$ (Mucciarelli et al. 2007). To explain the composition of the O and C-rich stars in NGC 1846, extra mixing was proposed to occur on the FGB and AGB, but only on the AGB once the stars had become carbon rich. In contrast, extra mixing on the AGB does not seem to be required in the LMC cluster NGC 1978, where the C-rich stars have high ${ }^{12} \mathrm{C} /{ }^{13} \mathrm{C}$ ratios $(\geq 150)$ (Lederer et al. 2009). Why the evolution of the C-rich stars in the cluster NGC 1978 should be so different to those in the cluster NGC 1846 is puzzling, given the small difference in metallicity. From the trend of the models in Fig. 4, we speculate that an intershell enhanced in both ${ }^{12} \mathrm{C}$ and ${ }^{16} \mathrm{O}$ may be able to explain the abundances of the LMC cluster NGC 1978. Stellar models of the appropriate mass and metallicity as the AGB stars in that cluster are needed to explore this possibility in more detail.

At lower metallicities $([\mathrm{Fe} / \mathrm{H}] \leq-2)$ very low ${ }^{12} \mathrm{C} /{ }^{13} \mathrm{C}$ ratios are measured in carbon-enhanced metal-poor (CEMP) stars (e.g., Beers \& Christlieb 2005; Sneden et al. 2008), as are high Lienrichments (Aoki et al. 2008; Thompson et al. 2008). The origin of the CEMP stars with enrichments of $s$-process elements has been suggested to be mass transfer from a previous AGB companion. This is supported by observations that suggest that CEMP stars with $s$-process elements are all members of binary systems (Lucatello et al. 2005). Extra mixing on the FGB of the 
donor AGB star would not be enough to explain the low carbon isotopic compositions measured in the CEMP stars (Sivarani et al. 2006; Campbell \& Lattanzio 2008), especially those that are still on the main sequence or sub-giant branch and whose ${ }^{12} \mathrm{C} /{ }^{13} \mathrm{C}$ ratios cannot therefore have been reduced by extra mixing during their own FGB. Stancliffe (2010) has suggested that thermohaline mixing can explain the Li enrichment, but the models do not have low enough ${ }^{12} \mathrm{C} /{ }^{13} \mathrm{C}$ ratios.

The oxygen isotope ratios measured in evolved giants also do not support a case for extra mixing on the AGB. The observed increase in the oxygen isotopic composition with evolutionary state can be explained by the action of the TDU and higher $\mathrm{O}$ intershell abundances than predicted by standard models (see also the discussion in Herwig 2000). Increasing $\mathrm{O}$ is motivated by both theoretical models of AGB stars as well as observations of hot post-AGB objects. One issue with this comparison are the large uncertainties in the measurements of oxygen isotope ratios in evolved stars. Updated measurements with smaller uncertainties would certainly help constrain this scenario. The oxygen isotope data for pre-solar oxide grains has been used as supporting evidence for the existence of efficient extra mixing on the FGB and AGB (Zinner et al. 2005; Nollett et al. 2003; Nittler et al. 1997; Wasserburg et al. 1995). However, the most extreme grains fall outside of the region determined for stellar spectra. If these grains have an AGB origin, then either they are formed in stars too dusty and heavily obscured for spectroscopic analysis, or the stars that form such grains are rare and not included in observational samples. One test that would help to confirm (or rule out) such mixing would be to derive accurate measurements of oxygen isotope ratios for large samples of O-rich and C-rich AGB stars covering a range of metallicities. This is desperately needed if we are to sort out the conflicting results for oxygen, especially given the consistency found for carbon between pre-solar SiC grains and AGB stars.

The models and tests we performed have many uncertainties, including the depth and efficiency of the third dredge-up, which may be stochastic in nature, as well as mass loss, opacities, and reaction rates. We have circumvented the uncertainties related to the origin of the extra mixing in FGB stars by adopting the observed abundance ratios at the tip of the FGB for our AGB models. We have also simply assumed that all stars experience extra mixing on the FGB such that the value of the ${ }^{12} \mathrm{C} /{ }^{13} \mathrm{C}$ ratio after mixing is equal to 10, whereas the observations by Gilroy \& Brown (e.g., 1991) show a variation between $\sim 10$ to 25 . Our results indicate that the observational data for $\mathrm{C} / \mathrm{O}$ and ${ }^{12} \mathrm{C} /{ }^{13} \mathrm{C}$ could in fact be fit better by assuming values between these extremes. Furthermore, the question of AGB intershell abundances may be affected by some of these uncertainties. Is convective overshoot the only way to obtain large O intershell abundances? Instead, could variations in key reaction rates (e.g., ${ }^{12} \mathrm{C}(\alpha, \gamma){ }^{16} \mathrm{O}$ ) allow us to synthesize more ${ }^{16} \mathrm{O}$ in the intershell? Clearly further investigation into the intershell abundances of AGB stars is warranted.

We thank the referee for many useful and instructive comments that have helped to improve this paper. AIK thanks Orsola De Marco for discussions about post-AGB stars, and acknowledges support from the Australian Research Council's Discovery Projects funding scheme (project number DP0664105), and is grateful for the support of the NCI National Facility at the ANU. 
This research was partially supported by a grant from the American Astronomical Society. SWC acknowledges the support of the Consejo Superior de Investigaciones Científicas (CSIC, Spain) JAE-DOC postdoctoral grant and the MICINN grant AYA2007-66256. RJS acknowledges support from the Australian Research Council's Discovery Projects funding scheme (project number DP0879472). 

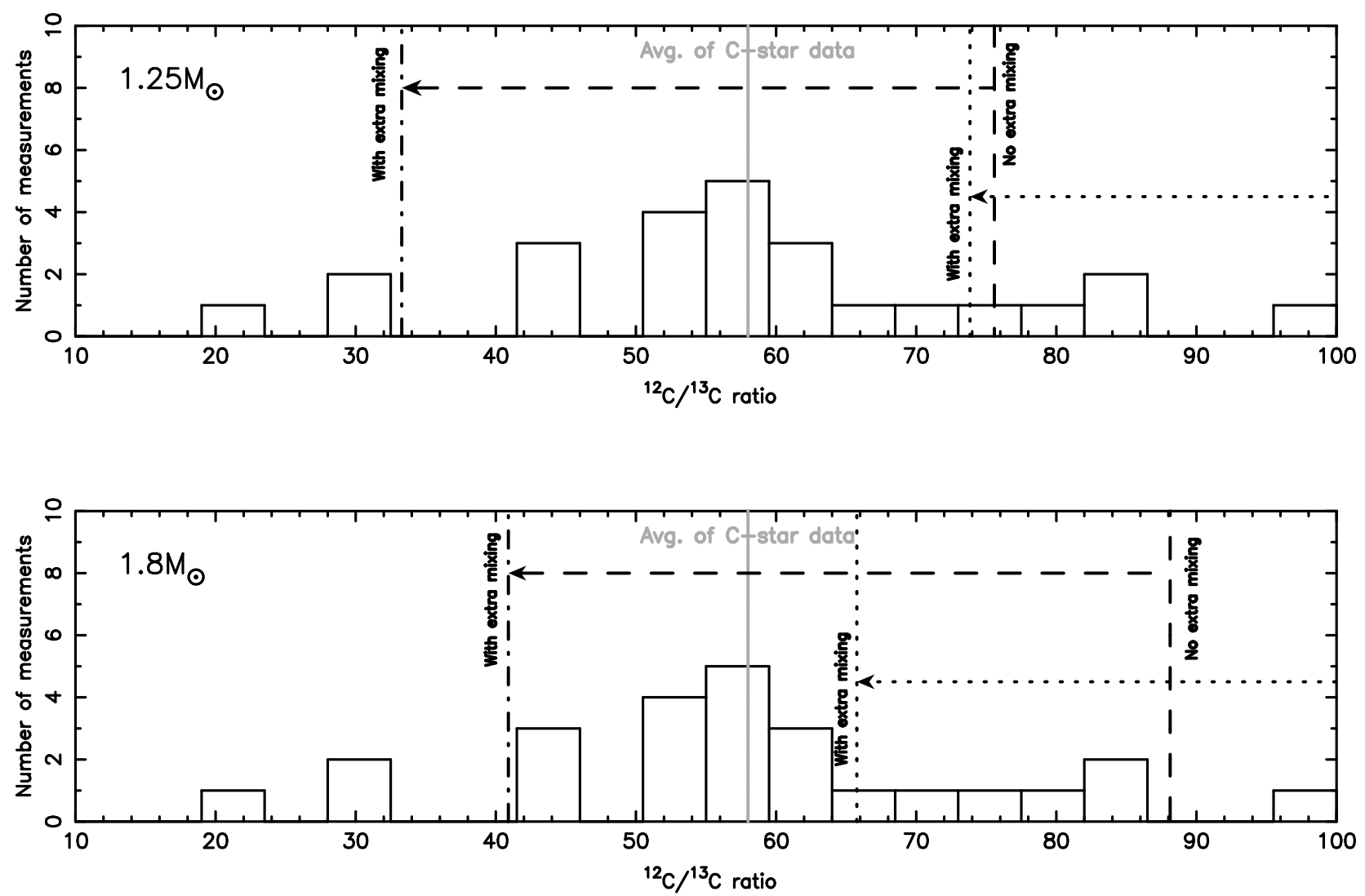

Fig. 1. - Histogram of observed ${ }^{12} \mathrm{C} /{ }^{13} \mathrm{C}$ ratios of AGB stars from Lambert et al. (1986). Data for the J-type carbon stars have been excluded. The average value (excluding the J-type stars) is 58, shown by the solid gray vertical line. With the J-stars, the average is 50 . The standard deviations are 25 and 17, with and without J-stars, respectively. The top panel shows model predictions from the $1.25 M_{\odot}$ model and the bottom panel for the $1.8 M_{\odot}$ case. In the top panel, the dashed vertical line shows the ${ }^{12} \mathrm{C} /{ }^{13} \mathrm{C}$ ratio at the tip of the AGB for the $N_{\text {ov }}=3$ model, and the dot-dashed vertical line shows the result when the ${ }^{12} \mathrm{C} /{ }^{13} \mathrm{C}$ ratio is reduced at the tip of the FGB. The dotted line shows the ratio for the $N_{\mathrm{ov}}=4$ model with extra mixing on the FGB. In the bottom panel, the dashed vertical line shows ${ }^{12} \mathrm{C} /{ }^{13} \mathrm{C}$ ratio at the tip of the AGB for the $N_{\text {ov }}=1$ model with no extra mixing, whereas the results including extra mixing are shown by the vertical dot-dashed line. The dotted line shows the ratio for the $N_{\text {ov }}=2$ with extra mixing on the FGB. 


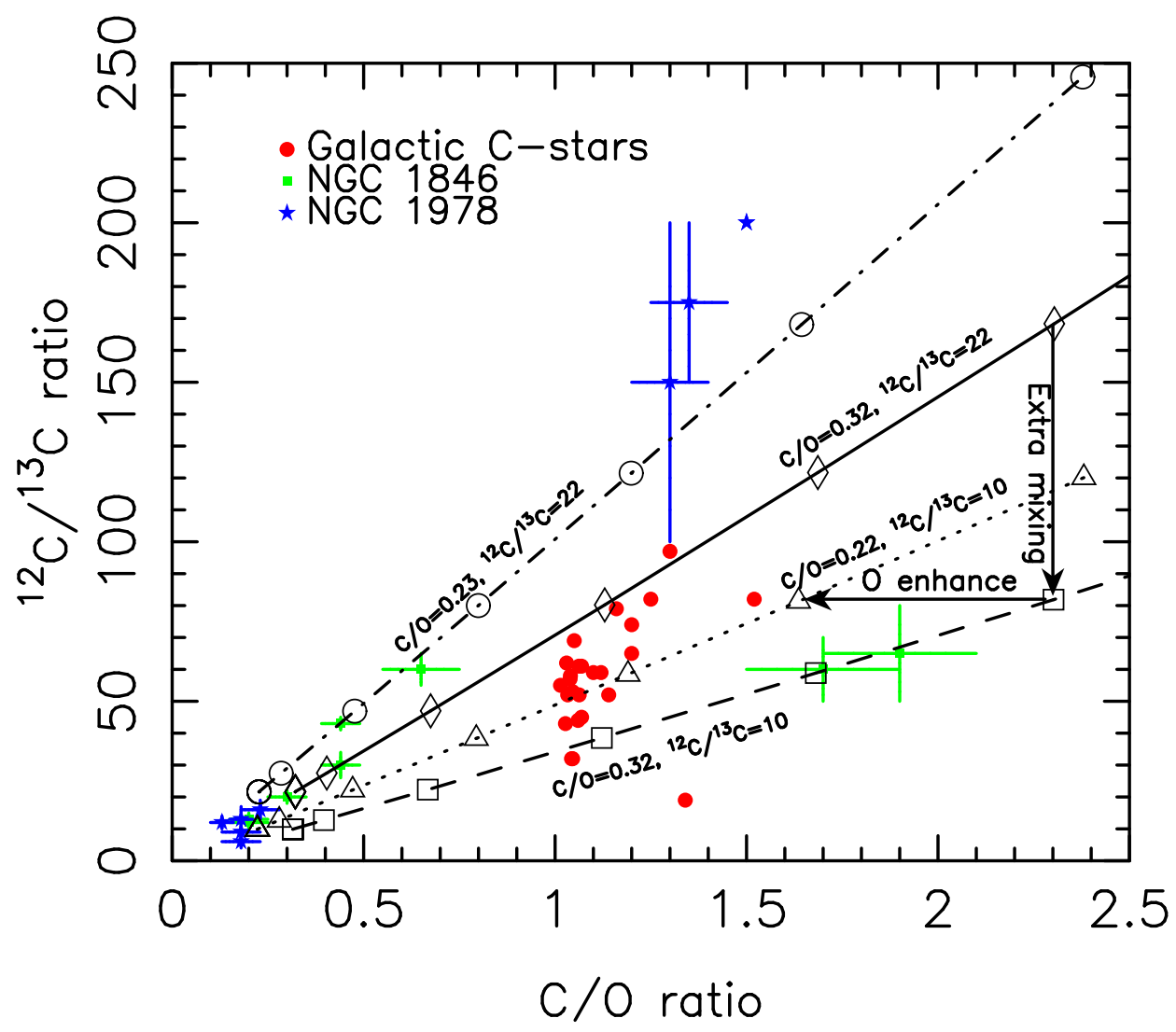

Fig. 2.- The $\mathrm{C} / \mathrm{O}$ ratio versus ${ }^{12} \mathrm{C} /{ }^{13} \mathrm{C}$. Observational data of Galactic C-stars from Lambert et al. (1986) are shown as solid circles, data for NGC 1846 from Lebzelter et al. (2008) as solid squares, and data for NGC 1978 from Lederer et al. (2009) as solid stars. Error bars are included for the two Magellanic Cloud clusters, except for one star which has a lower limit of 200 for ${ }^{12} \mathrm{C} /{ }^{13} \mathrm{C}$. Open symbols (connected by various line types) indicate model predictions from the $1.8 M_{\odot}$ model with $N_{\text {ov }}=3$ after each TDU episode. Predictions are shown for two models with a scaled solar composition, with and without extra mixing, and for two $\alpha$-enhanced models, with and without extra mixing. Labels indicate the $\mathrm{C} / \mathrm{O}$ and ${ }^{12} \mathrm{C} /{ }^{13} \mathrm{C}$ ratios at the tip of the $\mathrm{FGB}$, with the standard model indicated by the solid line. 


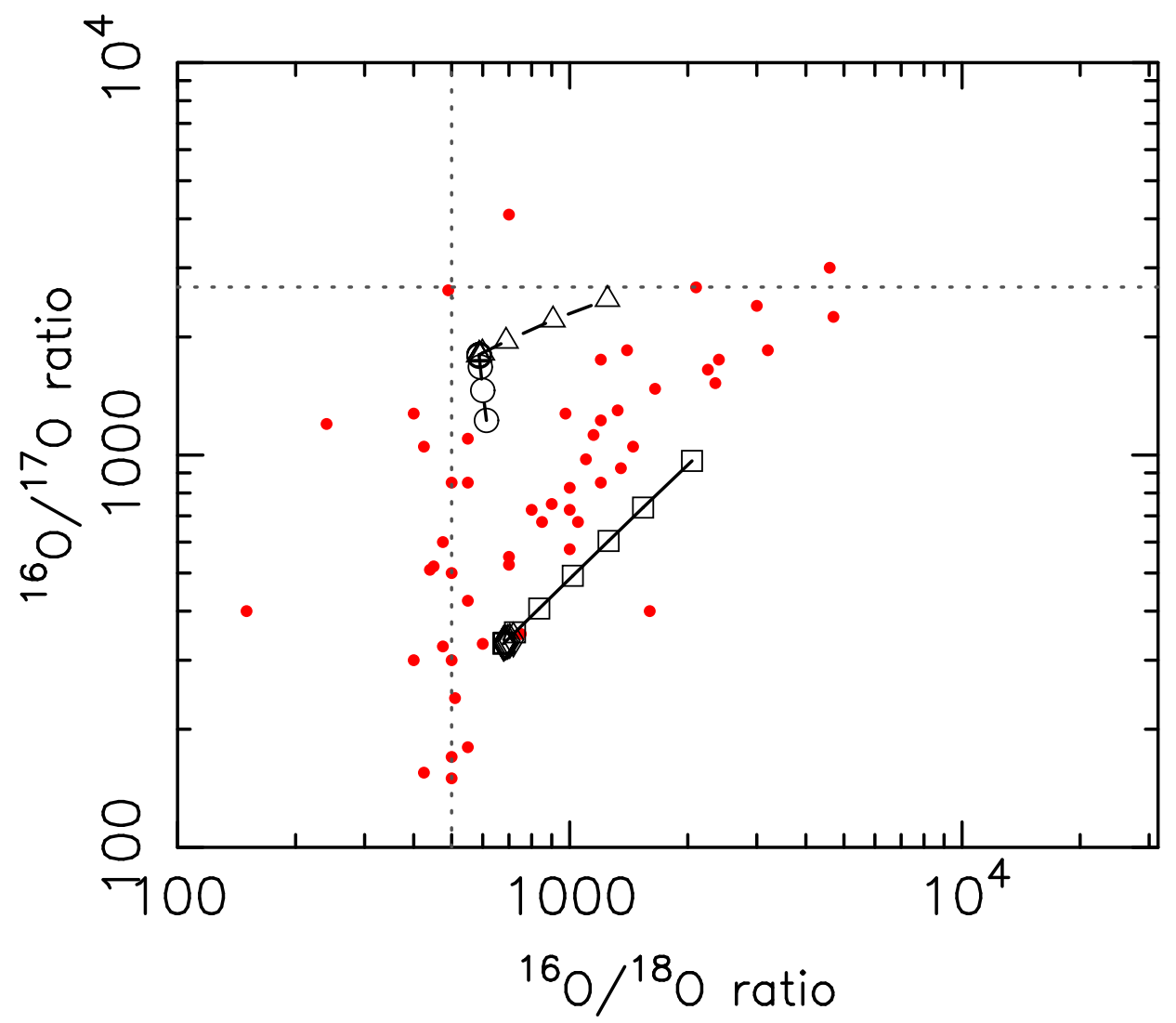

Fig. 3.- The ${ }^{16} \mathrm{O} /{ }^{18} \mathrm{O}$ ratio versus ${ }^{16} \mathrm{O} /{ }^{17} \mathrm{O}$. The solid points show observational data from Harris \& Lambert (1984), Harris et al. (1985a), Harris et al. (1985b), and Harris et al. (1987) for $\mathrm{G}$ and $\mathrm{K}$ giants, barium stars, MS and S-type stars, and carbon stars. Error bars are not shown but uncertainties are large; see e.g., Harris et al. (1987) for details. The dotted lines show the solar values for the oxygen isotope ratios, which are the initial values used in the stellar models. The open diamonds show the surface composition after each TDU from the $1.8 M_{\odot}$ model with $N_{\text {ov }}=3$ assuming a standard intershell composition, and open squares an ${ }^{16} \mathrm{O}$ intershell abundance of $20 \%$ (by mass). The open circles show results from the $1.25 M_{\odot}$ model with $N_{\text {ov }}=4$ assuming a standard intershell composition, and open triangles assuming an ${ }^{16} \mathrm{O}$ intershell abundance of $20 \%$. 


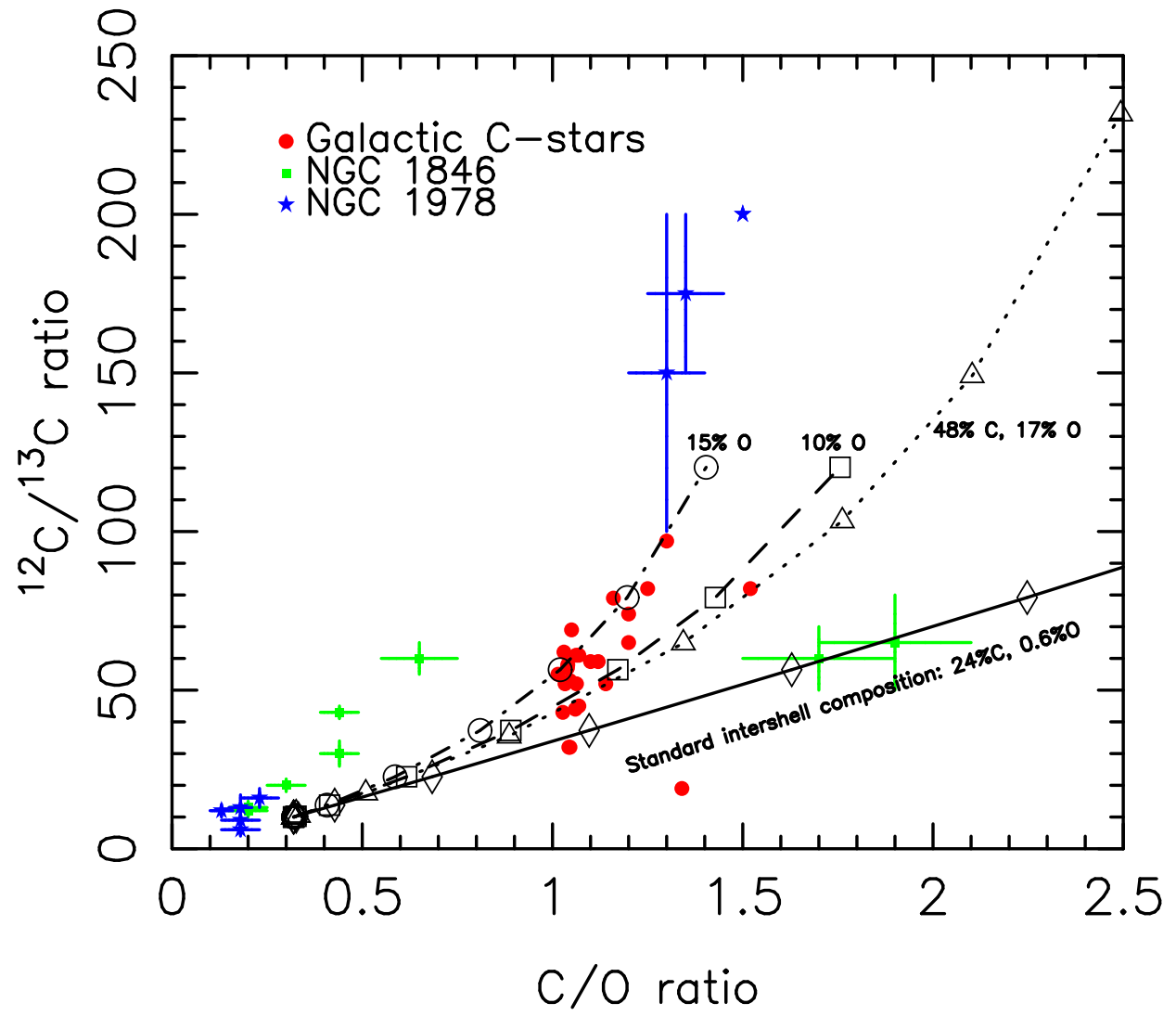

Fig. 4. - The $\mathrm{C} / \mathrm{O}$ ratio versus ${ }^{12} \mathrm{C} /{ }^{13} \mathrm{C}$. Filled symbols are the same as in Fig. 2, Open symbols indicate model predictions from the $1.8 M_{\odot}$ model with $N_{\text {ov }}=3$ with extra mixing on the FGB. In this plot we show the result of varying the intershell ${ }^{12} \mathrm{C}$ and ${ }^{16} \mathrm{O}$ abundances. The dotted line shows the result of using the composition of PG1159-035 which has $48 \% \mathrm{C}$ and $17 \% \mathrm{O}$. The dashed and dot-dashed lines shows the results of increasing the ${ }^{16} \mathrm{O}$ abundance from the standard $\approx 0.6 \%$ in the intershell to $10 \%$ (dashed) and $15 \%$ (dot-dashed), while keeping ${ }^{12} \mathrm{C}$ constant at $24 \%$. 
Table 1: Initial composition, post-FDU, tip-FGB for the extra-mixing case, and tip of the AGB abundance results for $\mathrm{C}, \mathrm{N}$, and $\mathrm{O}$.

\begin{tabular}{|c|c|c|c|c|c|c|c|c|}
\hline Position & $\bar{N}_{\mathrm{OV}}{ }^{\mathrm{a}}$ & $\operatorname{Mix}^{\mathrm{b}}$ & $\overline{\mathrm{C} / \mathrm{O}^{\mathrm{c}}}$ & ${ }^{112} \mathrm{C} /{ }^{13} \mathrm{C}$ & ${ }^{12} \mathrm{C} /{ }^{14} \mathrm{~N}$ & 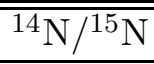 & 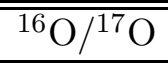 & 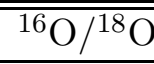 \\
\hline \multicolumn{9}{|c|}{$1.25 M_{\odot}, Z=0.01$ scaled-solar model. } \\
\hline Initial & - & - & 0.500 & 89.0 & 3.60 & 272.0 & 2680 & 500 \\
\hline post-FDU & - & - & 0.387 & 26.6 & 1.48 & 798.1 & 1801 & 587 \\
\hline tip-FGB & - & Yes & 0.335 & 10.0 & 1.00 & 960.2 & 1801 & 587 \\
\hline tip-AGB & 3.0 & No & 1.077 & 75.6 & 4.01 & 884.0 & 1240 & 610 \\
\hline tip-AGB & 3.0 & Yes & 1.015 & 33.3 & 3.13 & 1005 & 1229 & 607 \\
\hline tip-AGB & 4.0 & No & 2.213 & 160 & 8.38 & 876.9 & 1225 & 615 \\
\hline tip-AGB & 4.0 & Yes & 2.165 & 73.8 & 6.87 & 1039 & 1223 & 615 \\
\hline \multicolumn{9}{|c|}{$1.8 M_{\odot}, Z=0.01$ scaled-solar model. } \\
\hline Initial & - & - & 0.500 & 89.0 & 3.60 & 272.0 & 2680 & 500 \\
\hline post-FDU & - & - & 0.326 & 22.1 & 0.99 & 1269 & 331.0 & 678 \\
\hline tip-FGB & - & Yes & 0.320 & 10.0 & 0.91 & 1295 & 331.0 & 678 \\
\hline tip-AGB & 1.0 & No & 1.244 & 88.1 & 3.87 & 1309 & 325.8 & 694 \\
\hline tip-AGB & 1.0 & Yes & 1.252 & 40.9 & 3.66 & 1261 & 328.2 & 698 \\
\hline tip-AGB & 2.0 & No & 1.960 & 142 & 6.20 & 1292 & 331.0 & 703 \\
\hline tip-AGB & 2.0 & Yes & 1.966 & 65.8 & 6.31 & 1265 & 330.4 & 703 \\
\hline tip-AGB & 3.0 & No & 3.318 & 246 & 10.7 & 1217 & 338.9 & 717 \\
\hline tip-AGB & 3.0 & Yes & 3.318 & 120 & 10.4 & 1253 & 338.2 & 718 \\
\hline \multicolumn{9}{|c|}{$1.8 M_{\odot}, Z=0.01 \alpha$-enhanced model. } \\
\hline Initial & - & - & 0.350 & 89.0 & 3.60 & 272.0 & 3803 & 707 \\
\hline post-FDU & - & - & 0.230 & 22.1 & 0.99 & 1269 & 344.0 & 962 \\
\hline tip-FGB & - & Yes & 0.226 & 10.0 & 0.91 & 1293 & 344.0 & 962 \\
\hline tip-AGB & 3.0 & No & 2.378 & 246 & 10.7 & 1207 & 345.3 & 1000 \\
\hline tip-AGB & 3.0 & Yes & 2.380 & 120 & 10.4 & 1238 & 345.3 & 1000 \\
\hline
\end{tabular}

\footnotetext{
${ }^{a}$ This is the overshoot parameter that governs the efficiency of the TDU.

${ }^{b}$ This indicates if extra-mixing is assumed to occur on the first giant branch.

${ }^{c}$ All abundance ratios are by number.
} 
Table 2: AGB abundance results for $\mathrm{C}$ and $\mathrm{O}$ for models with various intershell compositions. The $1.25 M_{\odot}$ model has $N_{\text {ov }}=4$ while the $1.8 M_{\odot}$ model has $N_{\text {ov }}=3$.

\begin{tabular}{llrrrr}
\hline \hline${ }^{12} \mathrm{C}_{\text {is }}{ }^{a}$ & ${ }^{16} \mathrm{O}_{\text {is }}{ }^{a}$ & \multicolumn{1}{c}{$\mathrm{C} / \mathrm{O}^{b}$} & ${ }^{12} \mathrm{C} /{ }^{13} \mathrm{C}$ & ${ }^{16} \mathrm{O} /{ }^{17} \mathrm{O}$ & ${ }^{16} \mathrm{O} /{ }^{18} \mathrm{O}$ \\
\hline $0.26^{c}$ & $0.007 M_{\odot}, Z$ & 2.18 & 73.8 & 1225 & 615 \\
0.26 & 0.20 & 1.07 & 73.8 & 249 & 1248 \\
0.60 & 0.20 & 2.27 & 157 & 249 & 1248 \\
\hline \multicolumn{6}{c}{$1.8 M_{\odot}, Z=0.01$ scaled-solar model. } \\
\hline $0.24^{c}$ & $0.0058^{c}$ & 3.33 & 120 & 338 & 718 \\
$0.48^{d}$ & $0.17^{d}$ & 2.49 & 231 & 869 & 1843 \\
0.24 & 0.10 & 1.76 & 120 & 642 & 1364 \\
0.24 & 0.15 & 1.40 & 120 & 805 & 1706 \\
0.60 & 0.20 & 2.78 & 287 & 966 & 2049 \\
\hline \hline
\end{tabular}

${ }^{a}$ Intershell abundance (in mass fractions).

${ }^{b}$ All abundance ratios are by number.

${ }^{c}$ Standard intershell composition.

${ }^{d}$ The composition of the post-AGB star PG1159-035. 


\section{REFERENCES}

Abia, C., Boffin, H. M. J., Isern, J., \& Rebolo, R. 1993, A\&A, 272, 455

Abia, C., Busso, M., Gallino, R., Domínguez, I., Straniero, O., \& Isern, J. 2001, ApJ, 559, 1117

Abia, C., Domínguez, I., Gallino, R., Busso, M., Masera, S., Straniero, O., de Laverny, P., Plez, B., \& Isern, J. 2002, ApJ, 579, 817

Abia, C. \& Isern, J. 1997, MNRAS, 289, L11

Aoki, W., Beers, T. C., Sivarani, T., Marsteller, B., Lee, Y. S., Honda, S., Norris, J. E., Ryan, S. G., \& Carollo, D. 2008, ApJ, 678, 1351

Asplund, M., Grevesse, N., \& Sauval, A. J. 2005, in ASP Conf. Ser. 336: Cosmic Abundances as Records of Stellar Evolution and Nucleosynthesis, ed. T. G. Barnes, III \& F. N. Bash, 25

Beers, T. C. \& Christlieb, N. 2005, ARA\&A, 43, 531

Blöcker, T. 1995, A\&A, 297, 727

Boothroyd, A. I. \& Sackmann, I.-J. 1999, ApJ, 510, 232

Boothroyd, A. I., Sackmann, I.-J., \& Ahern, S. C. 1993, ApJ, 416, 762

Brown, J. A., Sneden, C., Lambert, D. L., \& Dutchover, E. J. 1989, ApJS, 71, 293

Busso, M., Wasserburg, G. J., Nollett, K. M., \& Calandra, A. 2007, ApJ, 671, 802

Campbell, S. W. \& Lattanzio, J. C. 2008, A\&A, 490, 769

Chafa, A., Tatischeff, V., Aguer, P., et al. 2007, Phys. Rev. C, 75, 035810

Charbonnel, C. 1994, A\&A, 282, 811

Charbonnel, C., Brown, J. A., \& Wallerstein, G. 1998, A\&A, 332, 204

Charbonnel, C. \& Zahn, J.-P. 2007, A\&A, 467, L15

De Marco, O. \& Barlow, M. J. 2001, Ap\&SS, 275, 53

Dearborn, D. S. P. 1992, Phys. Rep., 210, 367

Dearborn, D. S. P., Steigman, G., \& Tosi, M. 1996, ApJ, 465, 887

Denissenkov, P. A. \& Tout, C. A. 2000, MNRAS, 316, 395

Eggleton, P. P., Dearborn, D. S. P., \& Lattanzio, J. C. 2006, Science, 314, 1580

-. 2008, ApJ, 677, 581 
El Eid, M. F. 1994, A\&A, 285, 915

Forestini, M. \& Charbonnel, C. 1997, A\&AS, 123, 241

Frost, C. A. \& Lattanzio, J. C. 1996, ApJ, 473, 383

Gilroy, K. K. 1989, ApJ, 347, 835

Gilroy, K. K. \& Brown, J. A. 1991, ApJ, 371, 578

Grocholski, A. J., Cole, A. A., Sarajedini, A., Geisler, D., \& Smith, V. V. 2006, AJ, 132, 1630

Gyngard, F., Amari, S., Jadhav, M., Zinner, E., \& Lewis, R. S. 2006, in Lunar and Planetary Institute Science Conference Abstracts, Vol. 37, 37th Annual Lunar and Planetary Science Conference, ed. S. Mackwell \& E. Stansbery, 2194

Harris, M. J. \& Lambert, D. L. 1984, ApJ, 285, 674

Harris, M. J., Lambert, D. L., Hinkle, K. H., Gustafsson, B., \& Eriksson, K. 1987, ApJ, 316, 294

Harris, M. J., Lambert, D. L., \& Smith, V. V. 1985a, ApJ, 292, 620

-. 1985b, ApJ, 299, 375

Herwig, F. 2000, A\&A, 360, 952

-. 2005, ARA\&A, 43, 435

Jahn, D., Rauch, T., Reiff, E., Werner, K., Kruk, J. W., \& Herwig, F. 2007, A\&A, 462, 281

Kahane, C., Dufour, E., Busso, M., Gallino, R., Lugaro, M., Forestini, M., \& Straniero, O. 2000, A\&A, 357, 669

Karakas, A. I. 2010, MNRAS, in press, arXiv:0912.2142

Karakas, A. I., Lattanzio, J. C., \& Pols, O. R. 2002, Publ. Astron. Soc. Aust., 19, 515

Karakas, A. I., van Raai, M. A., Lugaro, M., Sterling, N. C., \& Dinerstein, H. L. 2009, ApJ, 690, 1130

Lambert, D. L., Gustafsson, B., Eriksson, K., \& Hinkle, K. H. 1986, ApJS, 62, 373

Lebzelter, T., Lederer, M. T., Cristallo, S., Hinkle, K. H., Straniero, O., \& Aringer, B. 2008, A\&A, 486,511

Lebzelter, T. \& Wood, P. R. 2007, A\&A, 475, 643

Lederer, M. T. \& Aringer, B. 2009, A\&A, 494, 403 
Lederer, M. T., Lebzelter, T., Cristallo, S., Straniero, O., Hinkle, K. H., \& Aringer, B. 2009, A\&A, 502,913

Lucatello, S., Gratton, R. G., Beers, T. C., \& Carretta, E. 2005, ApJ, 625, 833

Marigo, P. 2002, A\&A, 387, 507

Marigo, P., Girardi, L., \& Bressan, A. 1999, A\&A, 344, 123

Milam, S. N., Woolf, N. J., \& Ziurys, L. M. 2009, ApJ, 690, 837

Mowlavi, N. 1999, A\&A, 344, 617

Mucciarelli, A., Ferraro, F. R., Origlia, L., \& Fusi Pecci, F. 2007, AJ, 133, 2053

Nittler, L. R., Alexander, C. M. O., Gao, X., Walker, R. M., \& Zinner, E. 1997, ApJ, 483, 475

Nollett, K. M., Busso, M., \& Wasserburg, G. J. 2003, ApJ, 582, 1036

Nordhaus, J., Busso, M., Wasserburg, G. J., Blackman, E. G., \& Palmerini, S. 2008, ApJ, 684, L29

Palmerini, S., Busso, M., Maiorca, E., \& Guandalini, R. 2009, Publications of the Astronomical Society of Australia, 26, 161

Reddy, B. E., Tomkin, J., Lambert, D. L., \& Allende Prieto, C. 2003, MNRAS, 340, 304

Romano, D., Tosi, M., Matteucci, F., \& Chiappini, C. 2003, MNRAS, 346, 295

Sivarani, T., Beers, T. C., Bonifacio, P., et al. 2006, A\&A, 459, 125

Smith, G. H. 2002, PASP, 114, 1097

Smith, G. H. \& Tout, C. A. 1992, MNRAS, 256, 449

Sneden, C., Cowan, J. J., \& Gallino, R. 2008, ARA\&A, 46, 241

Stancliffe, R. J. 2010, MNRAS, in press, arXiv:0912.0762

Stancliffe, R. J., Church, R. P., Angelou, G. C., \& Lattanzio, J. C. 2009, MNRAS, 396, 2313

Stancliffe, R. J., Izzard, R. G., \& Tout, C. A. 2005, MNRAS, 356, L1

Stancliffe, R. J. \& Jeffery, C. S. 2007, MNRAS, 375, 1280

Stancliffe, R. J., Tout, C. A., \& Pols, O. R. 2004, MNRAS, 352, 984

Straniero, O., Chieffi, A., Limongi, M., Busso, M., Gallino, R., \& Arlandini, C. 1997, ApJ, 478, 332 
Thompson, I. B., Ivans, I. I., Bisterzo, S., Sneden, C., Gallino, R., Vauclair, S., Burley, G. S., Shectman, S. A., \& Preston, G. W. 2008, ApJ, 677, 556

Uttenthaler, S., Hron, J., Lebzelter, T., Busso, M., Palmerini, S., Schultheis, M., Käufl, H. U., Lederer, M. T., \& Aringer, B. 2008, A\&A, 478, 527

Uttenthaler, S. \& Lebzelter, T. 2010, A\&A, 510, 62

Vanture, A. D., Smith, V. V., Lutz, J., Wallerstein, G., Lambert, D., \& Gonzalez, G. 2007, PASP, 119,147

Vassiliadis, E. \& Wood, P. R. 1993, ApJ, 413, 641

Wallerstein, G. \& Knapp, G. R. 1998, ARA\&A, 36, 369

Wasserburg, G. J., Boothroyd, A. I., \& Sackmann, I.-J. 1995, ApJ, 447, L37

Werner, K., Rauch, T., Reiff, E., \& Kruk, J. W. 2009, Ap\&SS, 320, 159

Wesson, R., Barlow, M. J., Liu, X., Storey, P. J., Ercolano, B., \& de Marco, O. 2008, MNRAS, 383,1639

Zinner, E. 1998, Annual Review of Earth and Planetary Sciences, 26, 147

Zinner, E., Nittler, L. R., Hoppe, P., Gallino, R., Straniero, O., \& Alexander, C. M. O. . 2005, Geochim. Cosmochim. Acta, 69, 4149

This preprint was prepared with the AAS $\mathrm{LAT}_{\mathrm{E}} \mathrm{X}$ macros v5.2. 